\title{
A CLOSED GRAPH THEOREM
}

\author{
S. O. IYAHEN
}

ABSTRACT. A closed graph theorem is proved, implying that a Hausdorff locally convex space $E$ need not be barrelled if every closed linear map from $E$ into $F$ is continuous, where $F$ is a reflexive Fréchet or $L F$. space or a space of distributions.

A Hausdorff locally convex space $(E, \tau)$ is barrelled if and only if for each Banach space $(F, \xi)$, a closed linear map from $(E, \tau)$ into $(F, \xi)$ is necessarily continuous. This result of M. Mahowald [2, Theorem 2.2] is shown here (Corollary) to be false if instead $(F, \xi)$ is a reflexive Fréchet or $L F$-space.

Theorem. Let $(E, \tau)$ be a Hausdorff locally convex space. Suppose that for a family $\left(S_{a}\right)_{a \in \Phi}$ of absolutely convex compact subsets, there is no locally convex topology on $E$ strictly finer than $\tau$ which coincides with $\tau$ on each $S_{a}$. Let $(F, \xi)$ be a Hausdorff locally convex space in which every closed bounded set is compact. If every closed linear map from any Banach space into $(F, \xi)$ is bounded, then a closed linear map $f$ from $(E, \tau)$ into $(F, \xi)$ is necessarily continuous.

Proof. Let $E_{a}$ denote the linear span of $S_{a}$, and $\tau_{a}$ the $\tau$-induced topology on $E_{a}$. Let $\eta_{a}$ denote the Banach space topology on $E_{a}$ with the sequence $\left(S_{a} / n\right)_{n=1}^{\infty}$ of sets as a base of neighborhoods of the origin.

As the graph of the restriction map of $f$ to $E_{a}$ is closed in $\left(E_{\alpha}, \tau_{a}\right) \times$ $(F, \xi)$, it is necessarily closed in $\left(E_{\alpha}, \eta_{\alpha}\right) \times(F, \xi)$ since $\eta_{\alpha}$ is finer than $\tau_{a}$. By the hypothesis then, the closure $T_{a}$ of $f\left(S_{a}\right)$ in $(F, \xi)$ is compact. The graph $G_{a}$ of the restriction map $f_{a}$ of $f$ to $S_{a}$ is closed in the compact Hausdorff space $X_{\alpha}=S_{a} \times T_{a}$, where $S_{a}, T_{a}$ are considered under the $\tau$ - and $\xi$-induced topologies, respectively.

Let $P_{S_{a}}, P_{T^{\prime}}$ be the projection maps of $X_{a}$ onto $S_{a}, T_{a}$ respectively, and $P_{G_{a}}$ the restriction of $P_{S_{a}}$ to $G_{a}$. As $P_{G_{a}}$ is continuous and one-toone, it is necessarily a homeomorphism, since $G$ is compact. Therefore each map $f_{a}=P_{T_{a}} \circ P_{G_{a}}{ }^{-1}: S_{a} \rightarrow(F, \xi)$ is continuous. This implies that $f:(E, \tau) \rightarrow(F, \xi)$ is continuous.

In the Theorem, $(E, \tau)$ could be the dual of any complete Hausdorff locally convex space under the topology of compact convergence $[1, \mathrm{p} .16$,

Received by the editors September $11,1974$.

AMS (MOS) subject classifications (1970). Primary 46A30; Secondary 46A07.

Key words and phrases. Closed graph theorem, Montel and reflexive spaces. 
Example $B]$, and $(F, \xi)$ could be any Montel Fréchet or $L F$-space or any of the distribution spaces $\mathcal{E}, \mathcal{E}^{\prime}, \mathfrak{D}, \mathfrak{D}^{\prime}, \mathcal{T}, \mathcal{J}^{\prime}, \mathcal{O}_{M}, \mathcal{O}_{M}^{\prime}, \mathcal{O}_{c}, \mathcal{O}_{c}^{\prime}$ by [3].

Corollary. Let $(E, \tau)$ be the dual of a complete Hausdorff locally convex space under the Mackey topology $\tau$. If $(F, \xi)$ is a reflexive Fréchet or LFspace or any of the distribution spaces above, then any closed linear map $f$ from $(E, \tau)$ into $(F, \xi)$ is necessarily continuous.

Proof. Let $\tau_{1}$ denote the topology on $E$ of compact convergence and $\xi_{0}$ the weak topology on $F$ associated with $\xi$. The graph of $f$ is necessarily closed in $\left(E, \tau_{1}\right) \times\left(F, \xi_{0}\right)$, and by the Theorem then $f:\left(E, r_{1}\right) \rightarrow\left(F, \xi_{0}\right)$ is continuous. This implies that $f:(E, \tau) \rightarrow(F, \xi)$ is continuous.

\section{REFERENCES}

1. D. J. H. Garling, A generalized inductive limit topology for vector spaces, Proc. London Math. Soc. 14 (1964), 1-28. MR 28 \#4330.

2. M. Mahowald, Barrelled spaces and the closed graph theorem, J. London Math. Soc. 36 (1961), 108-110. MR 23 \#A2728.

3. L. Schwartz, Sur le théorème du graphe fermé, C. R. Acad. Sci. Paris Sér. A-B 263 (1966), A602-A605. MR 34 \#6494.

DEPARTMENT OF MATHEMATICS, UNIVERSITY OF BENIN, BENIN CITY, NIGERIA 